\title{
Diversidad, distribución espacio-temporal y turnos de vocalización de anuros (Amphibia, Anura) en un área ecotonal del nordeste de Argentina
}

\author{
Víctor H. Zaracho' \& Esteban O. Lavilla²
}

\author{
1. Laboratorio de Herpetología, Facultad de Ciencias Exactas y Naturales y Agrimensura, Universidad Nacional del Nordeste, Av. Libertad 5470, CP 3400 \\ Corrientes, Argentina. (victorzaracho@yahoo.com.ar) \\ 2. Fundación Miguel Lillo, Instituto de Herpetología, Miguel Lillo 251, CP 4000, San Miguel de Tucumán, Argentina. (eolavilla@gmail.com)
}

\begin{abstract}
Diversity, spatiotemporal distribution and calling daily time in anurans (Amphibia, Anura) of an ecotonal area in North-Eastern Argentina. Studies on diversity and natural history of anuran communities are basic to distinguish natural fluctuations from those due to human impacts (a key tool in conservation programs), to assess the role they play in ecosystems dynamics and to allow comparison of diversity gradients. With those objectives, this paper analyzes diversity, temporal and spatial distribution, and calling daily time of the anurans from three breeding sites at Esteros del Iberá, in northeastern Argentina. Samples were taken monthly, between July 2008 and June 2010. We recorded 26 species belonging to five families: Bufonidae (2), Hylidae (11), Leptodactylidae (11), Microhylidae (1) and Odontophrynidae (1). The study highlights variations in species richness per site, in the period, frequency and calling daily time and in the densities of calling males. The species richness is comparable to that of other similar wetlands, including the dominance of leptodactylids and hylid, a frequent pattern in Neotropical assemblages. The concentration of active species in the warmest and wettest months was associated with changes in temperature and rainfall, which were identified as important factors that initiate reproductive activity in seasonal environments.
\end{abstract}

KEYWORDS. Natural history, amphibians, advertisement calls, reproduction, Iberá.

\begin{abstract}
RESUMEN. Estudios acerca de la diversidad e historia natural de comunidades de anuros son elementales para distinguir entre fluctuaciones naturales de aquellas causadas por impactos antrópicos (herramienta clave en programas de conservación), evaluar el papel que juegan en la dinámica de los ecosistemas y permitir comparaciones de gradientes de diversidad. En este contexto, se analiza la diversidad, la distribución temporal y espacial, y los turnos de vocalización de anuros de tres sitios de reproducción en los Esteros del Iberá, en el nordeste de Argentina. Los muestreos fueron mensuales entre julio de 2008 y junio de 2010. Se registraron 26 especies pertenecientes a cinco familias: Bufonidae (2), Hylidae (11), Leptodactylidae (11), Microhylidae (1), y Odontophrynidae (1). Se encontraron variaciones en la riqueza de especies por sitio, en el periodo, frecuencia y turno de vocalización y en la abundancia de machos vocalizadores. La riqueza es similar al de otros humedales similares, incluyendo un importante dominio de leptodactílidos e hílidos, patrón frecuente en ensambles neotropicales. La concentración de las especies en actividad de vocalización en los meses más cálidos y lluviosos estuvo asociada con los cambios en la temperatura y en las precipitaciones, las cuales fueron señaladas como algunos de los factores que inician la actividad reproductiva en ambientes estacionales.
\end{abstract}

PALABRAS CLAVE. Historia natural, anfibios, cantos de anuncio, reproducción, Iberá.

En los últimos años se han dado a conocer importantes declinaciones y extinciones de anfibios, siendo uno de los grupos de vertebrados más sensibles a las alteraciones ambientales. Entre las posibles causas se señalan la destrucción de sus hábitats, enfermedades, el uso de contaminantes, el cambio climático, la presencia de especies invasoras y el comercio (Blaustein et al., 2011). Sin embargo, algunas poblaciones pueden presentar fluctuaciones naturales, dificultando la percepción de los impactos antrópicos (PeChMANn et al., 1991; Marsh, 2001). En este contexto, son necesarios programas de monitoreo a largo plazo (WILSON \& MARET, 2002) y conocimientos sobre el comportamiento y la ecología de las poblaciones de anuros (HodGKISON \& Hero, 2001), principalmente, en áreas con actividades antrópicas, que potencialmente pueden influir en estas fluctuaciones poblacionales. Ante la alarmante tasa de destrucción de los ecosistemas naturales, el estudio de ciclos reproductivos puede contribuir a entender la estructura de sus comunidades (BARBAULT, 1991). Un punto de partida pueden ser estudios tendientes a detectar épocas y turnos de reproducción de cada una de las especies que integran un ensamble. En la región Neotropical, la mayoría de estos estudios fueron realizados en ensambles del Bosque Atlántico, del Cerrado, o de zonas ecotonales entre estos ambientes (e.g., BorgES \& Juliano, 2007; Conte \& Rossa-Feres, 2007; Melo et al., 2007; SANTOS et al., 2007; ZINA et al., 2007; ForTI, 2009; NARVAes et al., 2009; GAREY \& da Silva, 2010; OdA et al., 2009; Kopp et al., 2010; CANelas \& BerToluci, 2007), y en menor número, en ensambles de Pampas y Amazonia (BERNARde, 2007; SANTos et al., 2008). Estudios de este tipo contribuyen en la evaluación del papel que los anuros juegan en la dinámica de los ecosistemas, además de permitir la comparación de gradientes de diversidad (Duellman, 1978; Duellman \& Koechlin, 1991). Adicionalmente, estos datos elementales facilitan futuros trabajo de monitoreo y manejo de poblaciones tendientes a mitigar la extinción de especies a nivel local.

En el nordeste argentino se encuentra uno de los humedales más grandes de Sudamérica, los Esteros del Iberá, en el que pueden encontrarse numerosos ambientes acuáticos como esteros, lagunas, bañados y cañadas, y 
ambientes terrestres como pastizales y bosques (NeIFF, 2004). Es considerada una región ecotonal en la que confluyen ambientes de pastizales y bosques hidrófilos. El hecho de que gran parte de su extensión está anegada permanentemente permitió limitar el avance del hombre moderno, de forma tal que el paisaje ha permanecido poco alterado hasta las últimas décadas del siglo XX (FUnDACIÓN BIOdiversidAD, 2004). Sin embargo, en sus márgenes, vastas extensiones de ambientes naturales, principalmente pastizales, han sido modificados por las actividades antrópicas, entre ellas la cría de ganado y plantaciones de arroz, sumado al avance de forestaciones que también han contribuido a la reducción de estos ambientes. Aunque es considerada reserva natural provincial, aproximadamente la mitad de su territorio es de dominio privado, con lo cual el control sobre los mismos es restringido, y la implementación de planes de manejo está limitada.

El objetivo de este trabajo fue describir la diversidad, la distribución temporal y espacial, y los turnos de vocalización de un ensamble de anuros en un área de transición de tres provincias biogeográficas con el fin de contribuir al conocimiento de la diversidad e historia natural de especies que habitan áreas abiertas, y establecer una línea de base para futuros planes de manejo.

\section{MATERIALES Y MÉTODOS}

El área de estudio se encuentra dentro de los límites de la Reserva Natural Provincial Esteros del Iberá, la cual ocupa unos $13.000 \mathrm{~km}^{2}$ del centro-norte de la provincia de Corrientes (Argentina). Es un área transicional de tres provincias fitogeográficas: Chaqueña, Paranaense y Espinal (CABRERA \& WiLlinK, 1980), las cuales confluyen a través de sus distritos Chaco Oriental, de los Campos y del Ñandubay respectivamente (CARNEvali, 2003). El patrón de paisajes, su origen, la química de sus aguas, la elevada riqueza de especies vegetales y animales, su estado prístino y su posición biogeográfica hacen de este humedal un sistema único en América (NeIFF \& PoI DE NeIFF, 2006).

El trabajo de campo se realizó en el sector suroeste de la Reserva, en la estancia Rincón del Socorro, 30 $\mathrm{km}$ al sur de la localidad de Colonia Carlos Pellegrini. En este sector dominado por ambientes de Espinal, las actividades agro-ganaderas fueron retiradas hace unos 10 años aproximadamente. Actualmente es una reserva privada, cuya superficie es destinada exclusivamente a fines de conservación. Con el fin de incluir diferentes lugares de reproducción, se seleccionaron tres sitios con características particulares, distantes unos $5 \mathrm{~km}$ entre sí:

Sitio 1. Borde del estero (28 $\left.37^{\prime} 2^{\prime \prime} \mathrm{S}, 57^{\circ} 25^{\prime} 38^{\prime \prime} \mathrm{W}\right)$ : corresponde a una franja inundable (bañado) de unos 300 $\mathrm{m}$ de ancho que se extiende entre los pirizales de Cyperus giganteus Vahl que crecen en el estero propiamente dicho, y una lomada arenosa cubierta por pastizales de Andropogon lateralis Nees. La disponibilidad de agua varía según el nivel del agua en el sistema, con épocas de sequías donde el agua está completamente retraída y el suelo queda completamente seco, hasta épocas donde el agua alcanza el borde del pastizal. Esto implica una importante variación de su profundidad y superficie de área anegada. Cuando el suelo está seco se observa una importante vegetación herbácea baja con algunas matas de Eryngium sp., a la que se suma una abundante vegetación flotante cuando está inundado (Fig. 1). El estrato arbustivo y arbóreo es escaso, con algunas palmeras y algunos árboles bajos. Dada la extensión de este sitio se seleccionó un área de $2500 \mathrm{~m}^{2}$, siempre lo más próximo al estero. Durante los muestreos, en cuatro ocasiones no se observaron espejos de agua.

Sitio 2. Laguna semipermanente (28 $39^{\prime} 52^{\prime \prime} \mathrm{S}$, $\left.57^{\circ} 21^{\prime} 43^{\prime \prime} \mathrm{W}\right)$ : con un área máxima de aproximadamente $1200 \mathrm{~m}^{2}$ y una profundidad media de aproximadamente $0.6 \mathrm{~m}$. Este sitio está rodeado por pastizales inundables (Andropogon lateralis), y en el cuerpo de agua hay abundante vegetación flotante y emergente, mientras que en su orilla crece un pastizal bastante denso con algunas hierbas, arbustos y árboles bajos (Fig. 2). En épocas de abundantes precipitaciones, el suelo de todo el área puede estar totalmente inundado. El área total examinada, incluyendo los márgenes, abarcó aproximadamente 2500 $\mathrm{m}^{2}$, incluyendo un radio en el área marginal de $30 \mathrm{~m}$. Durante los muestreos en seis ocasiones no se observaron espejos de agua.

Sitio 3. Charca temporaria $\left(28^{\circ} 41^{\prime} 18^{\prime \prime} \mathrm{S}\right.$, $\left.57^{\circ} 25^{\prime} 52^{\prime \prime} \mathrm{W}\right)$ : zona deprimida de aproximadamente 75 $\mathrm{m}^{2}$ de área y profundidad máxima de $0.3 \mathrm{~m}$. Todo el área, incluyendo la charca está cubierta por un pastizal (Andropogon lateralis), aunque en esta última el pastizal es más abierto, y ocasionalmente pueden observarse algunas plantas flotantes (Fig. 3). La disponibilidad de agua depende exclusivamente de las precipitaciones, y en 10 ocasiones estuvo completamente seca. El área total de muestreo incluyó una franja marginal de $50 \mathrm{~m}$ de ancho alrededor de la charca totalizando $2500 \mathrm{~m}^{2}$.

Los muestreos fueron limitados a los sitios de reproducción (SCOTT \& WoODWARD, 1994). Cada sitio fue muestreado mensualmente a lo largo de dos períodos anuales, desde julio de 2008 hasta junio de 2010, totalizando 72 noches de observaciones totales. Los sitios se visitaron generalmente los últimos días de cada mes, y cada muestreo se extendió desde las $17 \mathrm{~h}$ hasta la medianoche, excepto durante los primeros 6 meses que se extendieron hasta las $06 \mathrm{~h}$ (Ávila \& FerReIRA, 2004) con el fin de registrar los turnos de vocalización.

Para el inventario de especies se utilizó la técnica de relevamientos por encuentros visuales y acústicos limitados por tiempo (RöDEL \& ERNST, 2004). Diariamente, cada hora y durante 10 minutos eran registradas todas las especies vistas y/o oídas. Complementariamente, aquellas especies en actividad de vocalización, fueron consideradas para el estudio de los turnos de vocalización. La densidad poblacional de machos vocalizadores, con un rango subjetivo, se estimó usando los siguiente rangos: 1, para un individuo macho; 2 , para un coro de 2-5 machos; 3 , para un coro de 6-10 machos y 4 para un coro $>10$ machos (LIPS 

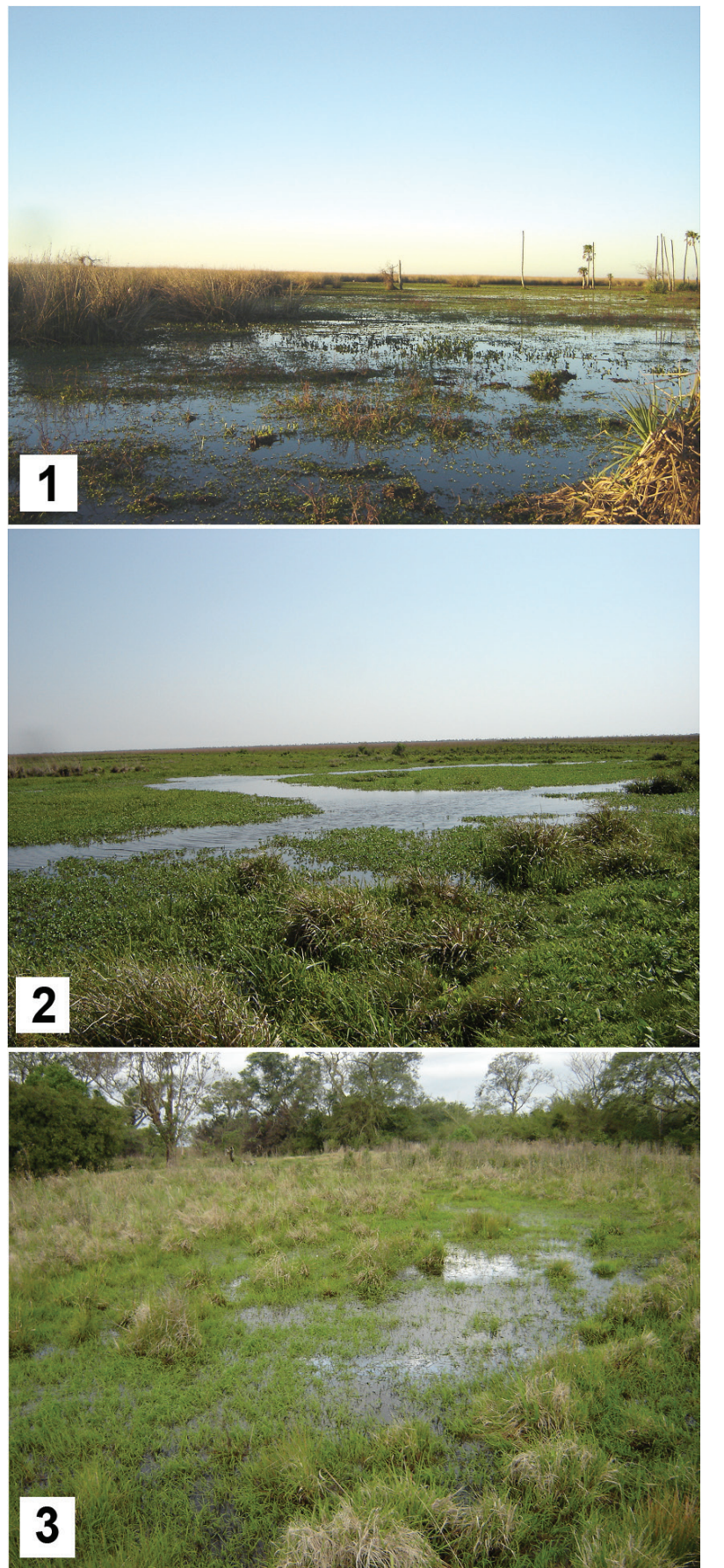

Figs 1-3. Cuerpos de agua donde fueron realizados los muestreos en la Reserva Natural Provincial Esteros del Iberá, Corrientes, Argentina: 1, borde de estero; 2 , laguna semipermanente; 3 , charca temporaria.

et al., 2001). La temperatura y la humedad media mensual, y las precipitaciones mensuales acumuladas se obtuvieron de una estación meteorológica Daza W1081 instalada en cercanías del Sitio 3. La temperatura y la humedad durante el momento de los muestreos se obtuvieron con un termohigrómetro digital TFA.

La diversidad alfa del área de estudio y de cada sitio se comparó mediante la riqueza (S) de especies. La riqueza esperada para el área y para cada sitio se estimó usando los estimadores de riqueza no paramétricos
Jackknife 2 (PALMER, 1990; KREBS, 1999), por contemplar heterogeneidad en las muestras y Chao 2 (СнAO, 1987; Colwell \& Coddington, 1994) sugerido como el más conveniente para estimar la riqueza basada en incidencia. A partir del valor obtenido se determinó la completitud del inventario, que fue expresado en porcentaje (\%). Estos análisis se realizaron mediante el reordenamiento aleatorio repetido de las muestras (1000 repeticiones), con el programa EstimateS versión v8.0.0 (http://purl.oclc.org/ estimates) (Colwell, 2009). La diversidad beta entre sitios se calculó mediante el índice de complementariedad (C) de Colwell \& Coddington (1994). Este índice varía entre 0 (sitios idénticos en composición) y 1 (sitios completamente distintos en composición). Los valores fueron expresados como el porcentaje de especies complementarias entre sitios (MORENo, 2001). Adicionalmente, se construyó una matriz de similitud usando el índice de Jaccard $\left(\mathrm{I}_{\mathrm{J}}\right)$, con la cual se realizó un análisis de clúster para determinar la similitud entre los sitios.

Se evaluó la influencia de las variables ambientales (temperatura, humedad y precipitaciones) en la riqueza diaria de especies en actividad de vocalización mediante un análisis de regresión múltiple, considerando a los tres sitios de muestreo como variables dummy (S1, S2 y S3). Adicionalmente, se calculó la importancia relativa de cada variable para la riqueza de especies utilizando el método LMG (LiNDEMANN et al., 1980) con un intervalo de confianza de $95 \%$ y 1000 réplicas de Bootstrap. Los valores diarios de temperatura y de humedad relativa del aire corresponden al valor promedio de tres mediciones realizadas en la franja horaria donde registramos el mayor número de especies vocalizando, y el de las precipitaciones, al total acumulado en los últimos 30 días previos a cada muestreo.

Los análisis estadísticos se realizaron utilizando la platoforma R versión 3.2.2. (R Core Team, 2015). Para la aplicación del método LGM se empleó el paquete relaimpo (GRÖMPING, 2006).

Los micro-ambientes de vocalización se determinaron a partir de observaciones directas de los adultos en actividad de vocalización, y los modos reproductivos fueron asignados según HADDAD \& PRADO (2005).

\section{RESULTADOS}

Se registraron 26 especies pertenecientes a 5 familias: Bufonidae (2), Hylidae (11), Leptodactylidae (11), Microhylidae (1) y Odontophrynidae (1) (Tab. I). Tres especies, Rhinella schneideri, Leptodactylus mystacinus and L. bufonius se registraron fuera de los sitios de muestreo, por lo que no fueron incluidos en los siguientes análisis. La completitud del inventario, según los estimadores empleados, fue de $86 \%$ y $96 \%$ para los tres sitios en conjunto, $71 \%$ y $81 \%$ para la charca temporaria, $82 \%$ y $94 \%$ para la laguna semipermanente y $85 \%$ y $89 \%$ para el borde del estero (Jackknife 2 y Chao 2 respectivamente). 
Para cada periodo de muestreo, la riqueza total fue mayor en el mes de febrero, con 17 y 16 especies respectivamente.

La riqueza de la charca temporaria y de la laguna semipermanente fue igual, con 21 especies (91\%). Sin embargo, en la primera no se registró Hypsiboas raniceps ni Leptodactylus podicipinus, mientras que en la última no se registró Argenteohyla siemersi pederseni ni Physalaemus albonotatus. Estas dos últimas especies fueron registradas únicamente en la charca temporaria. La riqueza del borde del estero fue $16(70 \%)$. Catorce especies $(61 \%)$ estuvieron presentes en todos los sitios, siete especies (30\%) en dos sitios, mientras que dos especies fueron exclusivas de un sitio (Tab. I).

Con respecto a la complementariedad entre sitios, fue mayor entre la charca temporaria y el borde del estero $(39 \%)$, mientras que entre el borde del estero y la laguna semipermanente fue $24 \%$, y entre la charca temporaria y la laguna semipermanente $17 \%$. En otros términos, como se observa en el análisis de cluster, la laguna y la charca son más similares en cuanto a la riqueza de especies (Fig. 4).

Todas las especies detectadas, excepto Leptodactylus chaquensis, se registraron con actividad de vocalización. Leptodactylus fuscus y Physalaemus albonotatus fueron registradas únicamente en el segundo periodo de muestreo.
La temporada de vocalización se extendió prácticamente a lo largo de todo el periodo de muestreo, con mayor riqueza en los meses de primavera y verano, y con picos en los meses de febrero (Tab. II). Únicamente en mayo de 2009 no se registró ninguna especie en actividad de vocalización, coincidiendo con un período marcado de sequía. Los picos de vocalizaciones se registraron entre las 19 y $22 \mathrm{~h}$.

Los turnos de vocalización, junto con la abundancia máxima por turno horario para cada especie, considerando los tres sitios en conjunto, se presentan en la Tabla III. En la Tabla I se incluyen las abundancias máximas de machos en actividad de vocalización y por especie para cada sitio, y en la Tabla II la frecuencia de especies en actividad de vocalización.

Las especies del ensamble mostraron variación en el periodo y frecuencia de vocalización a lo largo del año, en la cantidad de horas por día, en los tipos de sitios y en las densidades de machos vocalizadores por sitio (Tabs I-III).

Con respecto a los microambientes de vocalización, los leptodactílidos vocalizaron flotando en los cuerpos de agua (Physalaemus), sobre el suelo inundado (Pseudopaludicola falcipes) o fuera de los cuerpos de agua, a diferentes distancias de los mismos (Leptodactylus). La mayoría de los hílidos (Dendropsophus, Scinax, Hypsiboas

Tab. I. Lista de especies de anuros en un área del sector sureste de la Reserva Natural Provincial Esteros del Iberá (Corrientes, Argentina) registradas desde julio de 2008 hasta junio de 2010. Para cada especie se incluye el modo reproductivo (MR), la frecuencia total (\%) registrada en el área de estudio (FT) y la frecuencia (\%) de vocalización en cada sitio (entre paréntesis se incluye la densidad máxima relativa de machos vocalizadores: 1, un individuo macho; 2 , coro de 2-5 machos; 3 , coro de 6-10 machos y 4, coro > 10 machos; X: sin vocalizar). A: ausente (*, especies registradas ocasionalmente en el área, pero fuera de los sitios de muestreo).

\begin{tabular}{|c|c|c|c|c|c|}
\hline Especies & MR & FT & Charca & Laguna & Estero \\
\hline \multicolumn{6}{|l|}{ BUFONIDAE } \\
\hline Rhinella fernandezae (Gallardo, 1957) & 1 & 50 & $4(1)$ & $21(\mathrm{X})$ & $33(1)$ \\
\hline *Rhinella schneideri (Werner, 1894) & 1 & & & & \\
\hline \multicolumn{6}{|l|}{ HYLIDAE } \\
\hline Argenteohyla siemersi pederseni Williams \& Bosso, 1994 & 1 & 21 & $21(3)$ & A & A \\
\hline Dendropsophus nanus (Boulenger, 1889) & 1 & 58 & $33(3)$ & $42(3)$ & $54(3)$ \\
\hline Dendropsophus sanborni (Schmidt, 1944) & 1 & 63 & $25(3)$ & $46(4)$ & $54(4)$ \\
\hline Hypsiboas pulchellus (Duméril \& Bibron, 1841) & 1 & 79 & $29(2)$ & $42(4)$ & $71(4)$ \\
\hline Hypsiboas raniceps Cope, 1862 & 1 & 54 & A & $25(2)$ & $50(4)$ \\
\hline Pseudis limellum (Cope, 1862) & 1 & 46 & $4(X)$ & $33(4)$ & $42(4)$ \\
\hline Phyllomedusa azurea (Cope, 1862) & 24 & 42 & $38(2)$ & $33(2)$ & A \\
\hline Scinax berthae (Barrio, 1962) & 1 & 25 & $25(3)$ & $8(2)$ & A \\
\hline Scinax fuscomarginatus (A. Lutz, 1925) & 1 & 46 & $13(2)$ & $17(2)$ & $46(3)$ \\
\hline Scinax nasicus (Cope, 1862) & 1 & 21 & $13(2)$ & $17(3)$ & $4(1)$ \\
\hline Scinax squalirostris (A. Lutz, 1925) & 1 & 88 & $71(4)$ & $58(4)$ & $54(4)$ \\
\hline \multicolumn{6}{|l|}{ LEPTODACTYLIDAE } \\
\hline *Leptodactylus bufonius Boulenger, 1894 & 30 & & & & \\
\hline Leptodactylus chaquensis Cei, 1950 & 11 & 25 & $13(\mathrm{X})$ & $17(\mathrm{X})$ & A \\
\hline *Leptodactylus mystacinus (Burmeister, 1861) & 30 & & & & \\
\hline Leptodactylus fuscus (Schneider, 1799) & 30 & 13 & $8(2)$ & $4(\mathrm{X})$ & A \\
\hline Leptodactylus gracilis (Duméril \& Bibron, 1840) & 30 & 71 & $63(3)$ & $58(4)$ & $33(3)$ \\
\hline Leptodactylus latinasus Jiménez de la Espada, 1875 & 30 & 75 & $71(3)$ & $42(4)$ & $33(2)$ \\
\hline Leptodactylus latrans (Steffen, 1815) & 11 & 58 & $13(1)$ & $42(2)$ & $46(2)$ \\
\hline Leptodactylus podicipinus (Cope, 1862) & 13 & 13 & A & $4(3)$ & $13(3)$ \\
\hline Physalaemus albonotatus (Steindachner, 1864) & 11 & 4 & $4(1)$ & A & A \\
\hline Physalaemus riograndensis Milstead, 1960 & 11 & 29 & $8(1)$ & $25(3)$ & $4(1)$ \\
\hline Pseudopaludicola falcipes (Hensel, 1867) & 1 & 50 & $4(2)$ & $33(2)$ & $46(3)$ \\
\hline \multicolumn{6}{|l|}{ ODONTOPHRYNIDAE } \\
\hline Odontophrynus americanus (Duméril y Bibron, 1841) & 1 & 8 & $8(1)$ & $4(2)$ & A \\
\hline \multicolumn{6}{|l|}{ MICROHYLIDAE } \\
\hline Elachistocleis bicolor (Guérin-Meneville, 1838) & 1 & 21 & $13(4)$ & $13(2)$ & $8(2)$ \\
\hline Riqueza & & & 21 & 21 & 16 \\
\hline
\end{tabular}



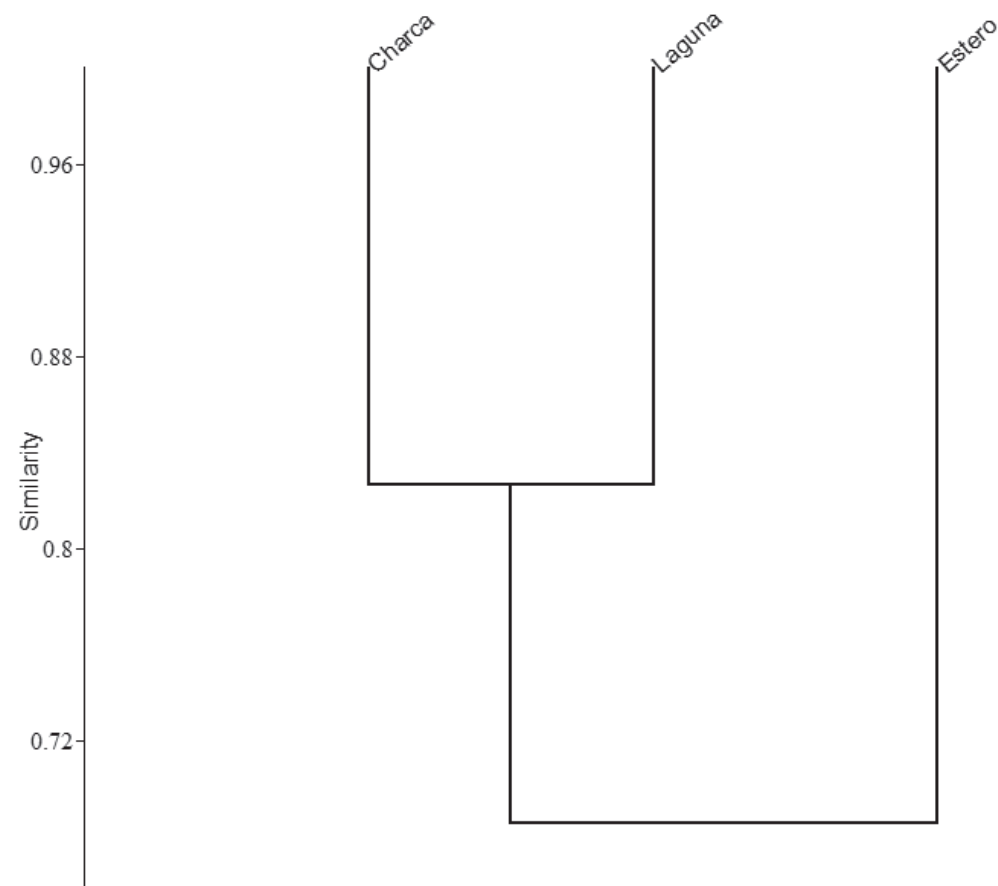

Fig. 4. Dendrograma agrupando los distintos sitios de reproducción de acuerdo a la similitud en la composición de especies (usando datos de presencia-ausencia), Reserva Natural Provincial Esteros del Iberá, Corrientes, Argentina.

Tab. II. Temporada y frecuencia de vocalización (FV) de anuros en un área del sector sureste de la Reserva Natural Provincial Esteros del Iberá (Corrientes, Argentina) desde julio de 2008 hasta junio de 2010.

\begin{tabular}{|c|c|c|c|c|c|c|c|c|c|c|c|c|c|c|c|c|c|c|c|c|c|c|c|c|c|c|}
\hline \multirow{2}{*}{ Especies } & \multicolumn{8}{|c|}{2008} & \multicolumn{9}{|c|}{2009} & \multicolumn{7}{|c|}{2010} & \multicolumn{2}{|c|}{$\mathrm{FV}$} \\
\hline & $\mathrm{J}$ & $\mathrm{A}$ & $\mathrm{S}$ & $\mathrm{O}$ & $\mathrm{N}$ & $\mathrm{D}$ & $\mathrm{E}$ & $\mathrm{F}$ & $\mathrm{M}$ & $\mathrm{A}$ & $\mathrm{M}$ & $\mathrm{J}$ & $\mathrm{J}$ & $\mathrm{A}$ & $\mathrm{S}$ & $\mathrm{O}$ & $\mathrm{N}$ & $\mathrm{D}$ & $\mathrm{E}$ & $\mathrm{F}$ & $\mathrm{M}$ & $\mathrm{A}$ & $\mathrm{N}$ & & $\mathrm{J}$ & $\%$ \\
\hline A.s. pederseni & & & & & & & & & & & & & & & & & & & & & & & & & & 17 \\
\hline D. nanus & & & & & & & & & & & & & & & & & & & & & & & & & & 50 \\
\hline D. sanborni & & & & & & & & & & & & & & & & & & & & & & & & & & 58 \\
\hline H. pulchellus & & & & & & & & & & & & & & & & & & & & & & & & & & 75 \\
\hline H. raniceps & & & & & & & & & & & & & & & & & & & & & & & & & & 50 \\
\hline S. berthae & & & & & & & & & & & & & & & & & & & & & & & & & & 25 \\
\hline S. fuscomarginatus & & & & & & & & & & & & & & & & & & & & & & & & & & 46 \\
\hline S. nasicus & & & & & & & & & & & & & & & & & & & & & & & & & & 21 \\
\hline S. squalirostris & & & & & & & & & & & & & & & & & & & & & & & & & & 79 \\
\hline P. limellum & & & & & & & & & & & & & & & & & & & & & & & & & & 42 \\
\hline P. azurea & & & & & & & & & & & & & & & & & & & & & & & & & & 42 \\
\hline L. fuscus & & & & & & & & & & & & & & & & & & & & & & & & & & 8 \\
\hline L. gracilis & & & & & & & & & & & & & & & & & & & & & & & & & & 71 \\
\hline L. latinasus & & & & & & & & & & & & & & & & & & & & & & & & & & 71 \\
\hline L. latrans & & & & & & & & & & & & & & & & & & & & & & & & & & 29 \\
\hline L. podicipinus & & & & & & & & & & & & & & & & & & & & & & & & & & 13 \\
\hline P. albonotatus & & & & & & & & & & & & & & & & & & & & & & & & & & 4 \\
\hline P. falcipes & & & & & & & & & & & & & & & & & & & & & & & & & & 38 \\
\hline P. riograndensis & & & & & & & & & & & & & & & & & & & & & & & & & & 25 \\
\hline R. fernandezae & & & & & & & & & & & & & & & & & & & & & & & & & & 13 \\
\hline O. americanus & & & & & & & & & & & & & & & & & & & & & & & & & & 4 \\
\hline E. bicolor & & & & & & & & & & & & & & & & & & & & & & & & & & 17 \\
\hline Total & 6 & 11 & 12 & 15 & 9 & 6 & 6 & 17 & 5 & 4 & 0 & 2 & 1 & 6 & 6 & 11 & 15 & 14 & 10 & 14 & 8 & 5 & 3 & & 5 & \\
\hline
\end{tabular}

y Phyllomedusa) por su parte, vocalizaron trepados a la vegetación emergente o marginal, a diferentes alturas; mientras que $A$. siemersi pederseni vocalizó flotando libremente en el cuerpo de agua y Pseudis limellum, apoyada sobre la vegetación flotante. Elachistocleis bicolor vocalizó desde el borde o interior de matas de vegetación inundada, apoyados sobre el suelo o la vegetación, con el cuerpo sumergido parcialmente. Rhinella fernandezae vocalizó flotando en los cuerpos de agua sostenido a la vegetación flotante mediante sus patas delanteras y Odontophrynus americanus desde el borde de los cuerpos de agua, sobre el suelo húmedo. 
Tab. III. Turnos de vocalización de anuros en un área del sector sureste de la Reserva Natural Provincial Esteros del Iberá (Corrientes, Argentina). Los diferentes tonos representan la densidad máxima de machos vocalizadores en esa franja horaria: 1, un individuo macho; 2 , coro de 2-5 machos; 3 , coro de 6-10 machos y 4 , coro $>10$ machos.

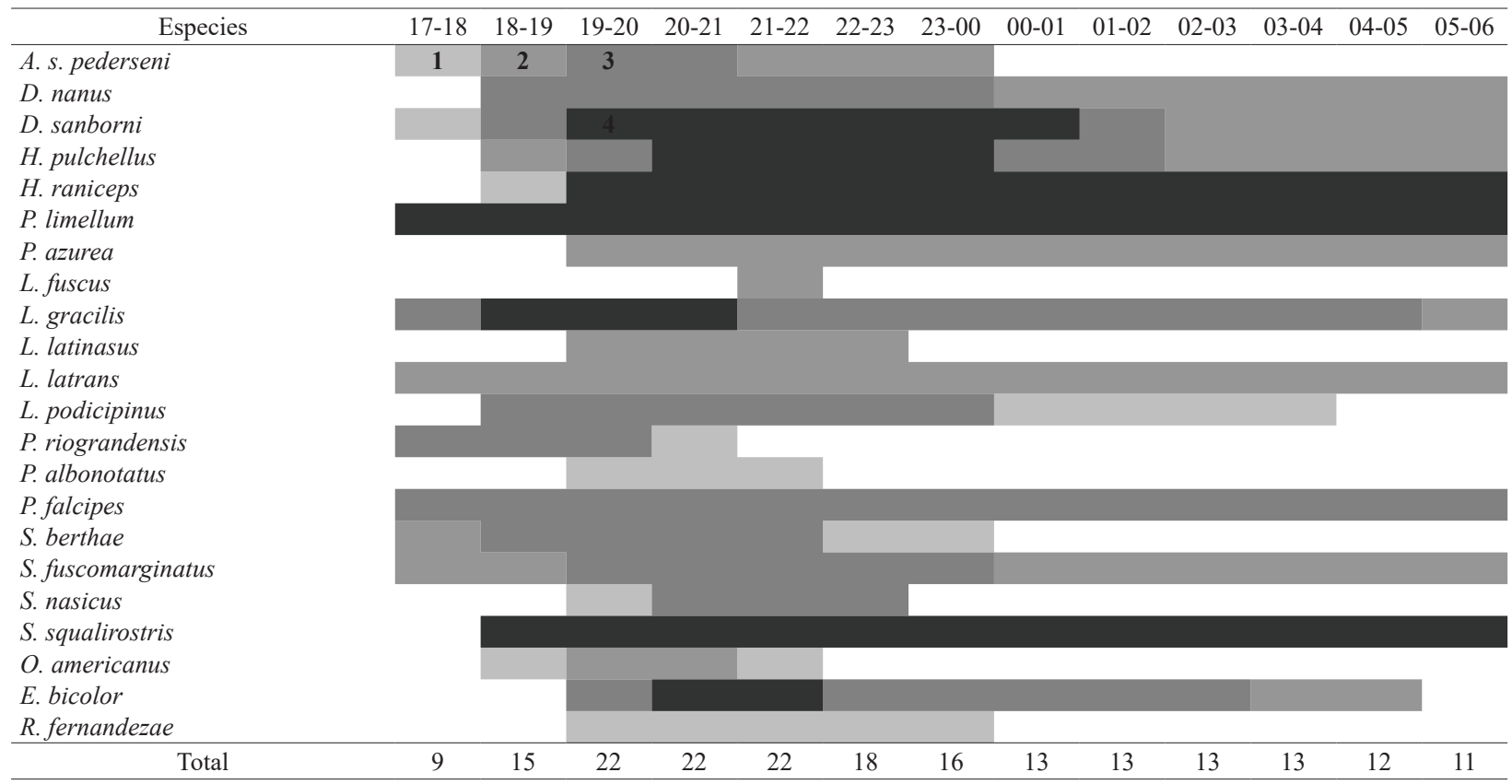

Las especies del ensamble presentan cinco modos reproductivos $(1,11,13,24,30)$, todos con fases larvales acuáticas obligadas.

La riqueza de especies total y en actividad de vocalización, la temperatura media mensual y las precipitaciones mensuales a lo largo del período de estudio se presentan en la Figura 5. En el análisis de regresión múltiple entre la riqueza diaria de especies vocalizando y las variables ambientales, incluyendo a los sitios como una variable dummy (y al S3 como referencia por poseer la riqueza más baja) no se observaron diferencias significativas entre los sitios ( $\mathrm{S} 2, \mathrm{t}=0,471, \mathrm{p}=0,639 ; \mathrm{S} 1, \mathrm{t}=0,899, \mathrm{p}=0,372$;

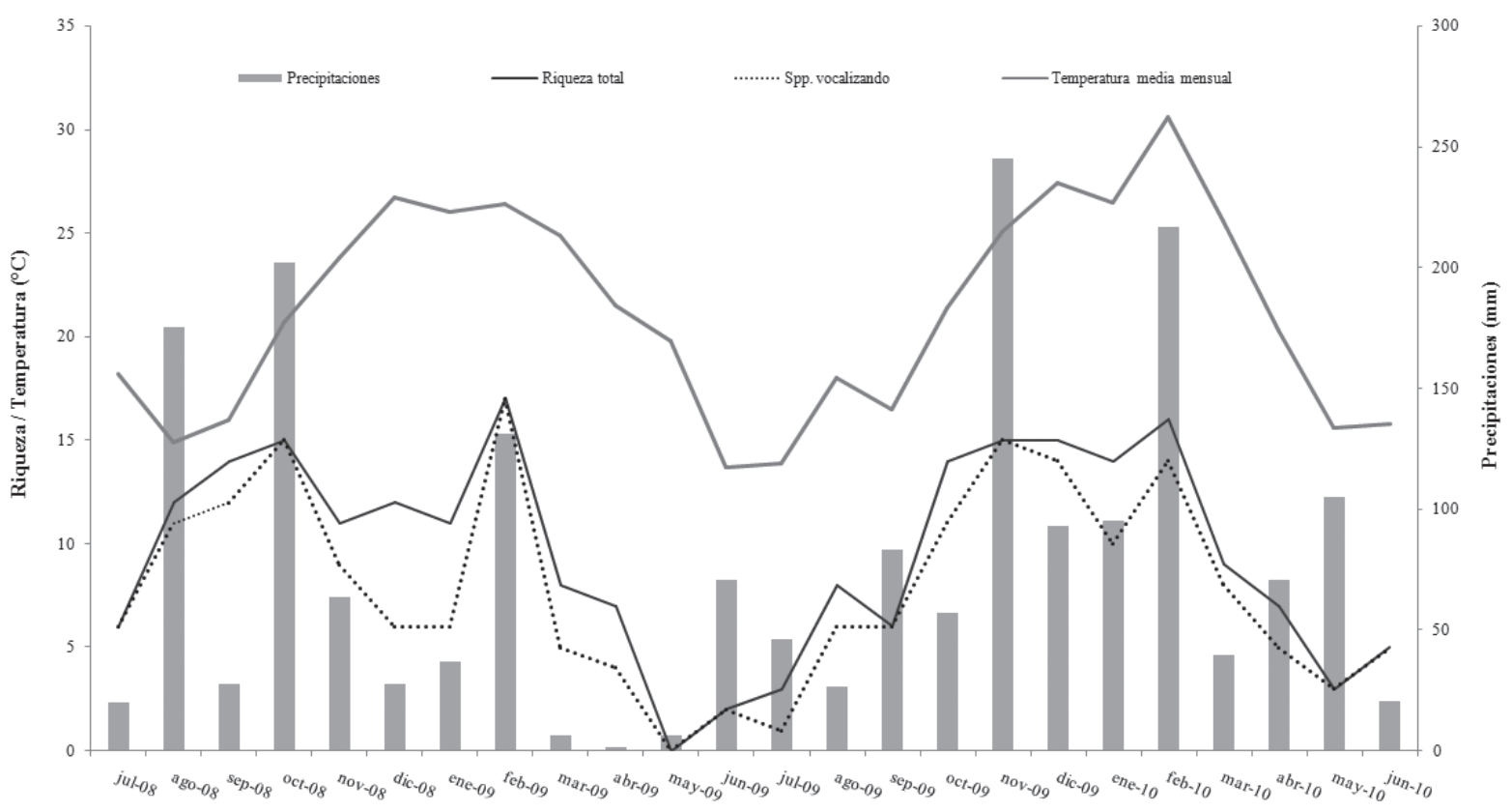

Meses

Fig. 5. Riqueza de especies de anuros total y en actividad de vocalización, en relación a las precipitaciones mensuales acumuladas y temperatura media mensual en un área del sector sureste de la Reserva Natural Provincial Esteros del Iberá, Corrientes, Argentina. 
$\mathrm{n}=72$ ). Por lo tanto, los sitios fueron considerados como réplicas y se realizó una nueva regresión múltiple utilizando los valores diarios promedios de temperatura y humedad de cada sitio y la riqueza diaria acumulada. Este análisis mostró una relación con las precipitaciones y con la temperatura, pero no con la humedad $\left(\mathrm{R}^{2}\right.$ ajustado $=0,69$; precipitaciones, $t=4,427, p=0,000$; temperatura: $t=4,330$, $\mathrm{p}=0,003$; humedad: $\mathrm{t}=1,68, \mathrm{p}=0,109, \mathrm{n}=24)$. Con respecto a la importancia relativa de cada variable en la riqueza de especies vocalizando, según el método LMG (basado en 24 observaciones), la proporción de la varianza explicada por el modelo fue de $73,17 \%$. Las contribuciones relativas de las precipitaciones, la temperatura y la humedad fueron $53,1 \%, 40,6 \%$ y $6,2 \%$ respectivamente (Fig. 6).

\section{Relative importances for Riqueza with $95 \%$ bootstrap confidence intervals}

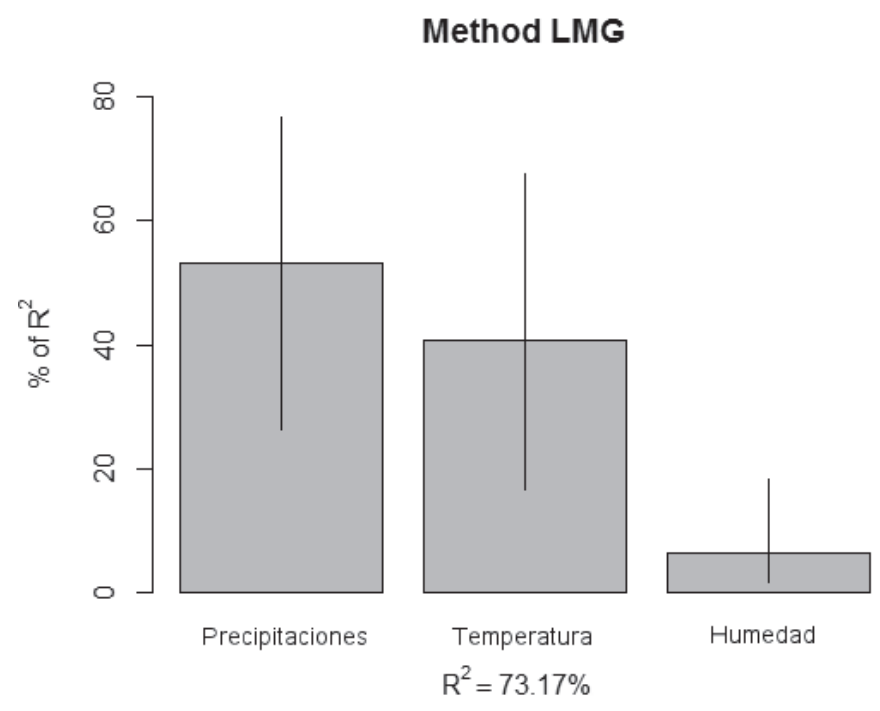

Fig. 6. Importancia relativa de cada variable ambiental para la riqueza de especies vocalizando, en un sector de la Reserva Natural Provincial Esteros del Iberá, Corrientes, Argentina.

\section{DISCUSIÓN}

Las 26 especies halladas en este estudio representan aproximadamente el $65 \%$ de las especies conocidas para la Reserva Natural Provincial Esteros del Iberá y casi un cuarto de las especies de anuros del nordeste argentino (Ingaramo et al., 2012; Vaira et al., 2012). Para una localidad cercana (a unos $30 \mathrm{~km}$ ) se registraron previamente 22 especies, entre las cuales se incluyó a Pseudis platensis, no registrada en este trabajo (Ingaramo et al., 2012). Aquí, se adicionan Scinax fuscomarginatus, Leptodactylus bufonius, L. fuscus, L. mystacinus y Argenteohyla siemersi pederseni, con lo cual la riqueza para el extremo sureste de la región asciende a 27 especies. Para el extremo norte de la reserva (a unos $140 \mathrm{~km}$ ) recientemente se citaron 30 especies, 21 de ellas en común con este estudio (INGARAMO et al., 2012; ZARACHO et al., 2014).

Si bien en la región no existen especies endémicas, su importancia radica en el hecho de que contiene representantes de diferentes provincias fitogeográficas. Esta riqueza, además, está incluida en el rango de lo conocido para otras localidades de humedales similares, como el Pantanal Brasileño, donde se registraron entre
19 y 37 especies, de las cuales aproximadamente el 50\% de esas son compartidas con las de este estudio (Ávila \& Ferreira, 2004; Gordo \& Campos, 2005; Prado et al., 2005; Uetanabaro et al., 2007; Pansonato et al., 2011).

La dominancia de especies de Leptodactylidae e Hylidae fue señalado como un patrón frecuente en ensambles neotropicales (Duellman, 1999; Pansonato et al., 2011). Sin embargo, las proporciones en la que están presentes son variables. El número de especies para ambas familias son similares y/o dominantes sobre otras, principalmente en ambientes de áreas más abiertas, en coincidencia con el de localidades situadas en las provincias biogeográficas del Cerrado (e.g., Araujo et al., 2009; Oda et al., 2009; Kopp et al., 2010; Pansonato et al., 2011; Gambale et al., 2014; Sugai et al., 2014), Caatinga (ArZabe, 1999; VieIra et al., 2007), Chaco (Perotti, 1997; ZARACHO et al., 2014), y Pampas (SANTOS et al., 2008). En ambientes más cerrados, como en el Bosque Atlántico, existe un marcado predominio de hílidos (e.g., ForTI, 2009; Narvaes et al., 2009; GAREY \& DA Silva, 2010; Martins et al., 2014). Los leptodactílidos se ven favorecidos por la mayor distribución horizontal de los microambientes de las áreas abiertas, principalmente aquellas con diferentes tipos 
de ambientes acuáticos (CARDoso et al., 1989). Por su parte, si bien los hílidos son favorecidos por la estratificación vertical de la vegetación, como en bosques y selvas, la vegetación emergente en áreas abiertas como el de este estudio también contribuye a una mayor diversidad de microambientes, y por lo tanto, a la riqueza de hílidos (CARdoso et al., 1989; Prado \& Pombal, 2005).

La duración del periodo reproductivo en los anuros puede enmarcarse entre dos patrones temporales claramente reconocidos: prolongado y explosivo (WeLL, 1977). En regiones tropicales y subtropicales, las condiciones ambientales son favorables por varios meses, y por lo tanto, muchas especies se reproducen más o menos continuamente a lo largo de toda la estación lluviosa. Otras especies, sin embargo, forman agregaciones explosivas después de fuertes lluvias. En este trabajo evitamos asignar estos patrones a las especies del ensamble pues no siempre las vocalizaciones están relacionadas con la actividad reproductiva, y se requieren de observaciones adicionales como hembras grávidas, amplexos y puestas.

La disponibilidad de agua en el borde del estero y en la laguna semipermanente al comienzo de la temporada reproductiva permite la presencia de sitios de reproducción, independientemente de las primeras precipitaciones de la temporada reproductiva, que sí son fundamentales para acumular agua en las charcas, y por lo tanto favorecer a aquellas especies que utilizan preferentemente estos sitios, como Argenteohyla siemersi pederseni o Physalaemus albonotatus, y posiblemente también a otras especies con patrón reproductivo explosivo como Elachistocleis bicolor, Rhinella fernandezae y Scinax nasicus (PRADo et al., 2005; CAJADE et al., 2010). Cuando las precipitaciones son insuficientes para formar los cuerpos de agua temporarios, algunas especies comunes en las charcas como Scinax squalirostris, Phyllomedusa azurea, Leptodactylus latinasus y Scinax berthae, pueden continuar vocalizando en las lagunas semipermanentes.

La diversidad de modos reproductivos es una de las características más destacadas entre los anuros (HADDAD \& Prado, 2005). Considerando que cualquiera de los cinco tipos de modos reproductivos que presentan las especies del ensamble estudiado incluyen una fase larval acuática obligada, la disponibilidad de agua en los sitios es elemental para la reproducción de las especies. Este requerimiento se ve favorecido por las características geográficas de la región, ya que aproximadamente más del 50\% de su superficie permanece inundada permanentemente, y en sus márgenes existen abundantes lagunas, esteros, bañados y charcas que permiten la disponibilidad de numerosos sitios de reproducción. Sin embargo, debe tenerse en cuenta que el sistema se abastece únicamente a partir de precipitaciones locales.

La relación de los factores abióticos como la temperatura, las precipitaciones y la humedad relativa del aire en la riqueza de especies en actividad de vocalización han sido previamente analizados en algunos ensambles neotropicales.
Relaciones positivas entre la riqueza de especies con las precipitaciones y la temperatura, como las encontradas en este trabajo, han sido previamente mencionadas en diferentes ensambles de anuros (Toledo et al., 2003; Prado et al., 2005; VASCONCELOS \& RosSA-FERES, 2005; Borges \& Juliano, 2007; CANElas \& Bertoluci, 2007; Melo et al., 2007; ZINA et al., 2007; SANTOs et al., 2007, 2008 ; ODA et al., 2009). Asimismo, la concentración de las especies en actividad de vocalización en los meses más cálidos y lluviosos está asociado a los cambios en la temperatura y las precipitaciones, que fueron señalados como algunos de los factores que inician la actividad reproductiva de los anfibios en ambientes estacionales (Duellman, 1978). Sin embargo, la influencia de otros factores como la luminosidad, el viento, y factores intrínsecos del área de estudio, también pueden influir sobre esta actividad, pero han sido escasamente abordados (Bellis, 1962; Toledo et al., 2003; Prado \& Pombal, 2005). En un ensamble de anuros en el Sur de Brasil por ejemplo, y a la misma latitud que el ensamble estudiado en este trabajo, la riqueza y abundancia de anuros vocalizando fue explicado únicamente por el fotoperiodo, si bien este factor estuvo cercanamente correlacionado con la temperatura máxima; la ausencia de correlación con las precipitaciones fue explicada por su distribución a lo largo de todo el año, las cuales no determinan ningún tipo de estacionalidad (Bотн et al., 2008).

De acuerdo a nuestros resultados, las precipitaciones fueron uno de los factores ambientales que más relación tuvo con la riqueza de especie. Posiblemente, en sitios como el de este trabajo, donde pueden alternarse años con abundantes precipitaciones con años de sequías marcadas, las precipitaciones se convierten en un factor determinante para la reproducción de los anuros, pues si bien aunque el fotoperiodo y la temperatura pueden ser las adecuadas para la reproducción, la ausencia o escasez de precipitaciones reduce la presencia de cuerpos de agua, y por consiguiente, de sitios de reproducción. De esta manera, las condiciones ambientales de un determinado lugar juegan un rol fundamental en la dinámica de sus ensambles.

El área de estudio comprende una zona de transición entre los distritos Chaco Oriental, del Ñandubay y de los Campos (correspondiendo estos últimos, principalmente, a ambientes abiertos de pastizal). Este trabajo es pionero para el área, ya que la mayoría de los estudios que abordan distribución espacio-temporal (y en menor medida turnos de vocalización) de ensambles de anuros neotropicales fueron realizados principalmente en áreas de Bosque Atlántico (e.g., Conte \& Rossa-Feres, 2007; SAntos et al., 2007; ZINA et al., 2007; ForTI, 2009; NARVAES et al., 2009; GAREY \& DA Silva, 2010), Cerrado (e.g., Melo et al., 2007; ODA et al., 2009; Kopp et al., 2010), Caatinga (ArzaBe, 1999; VieIRA et al., 2007) y Amazonia (BERNARde, 2007); o bien en áreas transicionales como por ejemplo entre Cerrado y Bosque Atlántico (Bernarde \& KoKubum, 1999; GrandinetTi \& JACOBI, 2005; VASCONCELOS \& Rossa-Feres, 2005; Borges \& Juliano, 2007; CANELAS \& 
Bertoluci, 2007) o entre Amazonia y Cerrado (SCHUlze et al., 2009). Estudios previos en Argentina fueron realizados en un relicto austral de Selva Paranaense (BASso, 1990), en el Chaco semiárido (Perotti, 1997), y selvas de las Yungas (VAIRA, 2002).

Finalmente, el área de la Reserva Natural Provincial Esteros del Iberá incluye, casi en las mismas proporciones, territorios de dominio público y privado. Aunque algunos territorios de dominio privado tienen una protección efectiva, y están actualmente destinadas a conservación, en el resto de la región, aún se realizan prácticas agro-ganaderas y forestales sin control, por lo que la contaminación del agua por el uso intensivo de agroquímicos o la pérdida de hábitats nativos por expansión de la frontera forestal representan una seria amenaza para la conservación de los anfibios de este vasto ecosistema. Ante este panorama, el conocimiento de la anurofauna local, mediante estudios como el aquí abordado, son elementales para la aplicación de planes de manejo que regulen estas actividades en los diferentes tipos de ambientes acuáticos de la región.

Agradecimientos. A dos revisores anónimos cuyas sugerencias contribuyeron a mejorar este manuscrito. A K. Facure y A. Giaretta por su colaboración con los análisis estadísticos. A la Dirección de Recursos Naturales de la Provincia de Corrientes (Argentina) por otorgarnos los permisos de investigación en áreas de la reserva natural provincia Esteros del Iberá. A Conservation Land Trust (CLT) y su personal por permitirnos realizar este trabajo en la Estancia Rincón del Socorro y brindarnos apoyo logístico durante los muestreos. A mis compañeros del Laboratorio de Herpetología por el acompañamiento en las salidas de campo. Al Consejo Nacional de Investigaciones Científicas y Técnicas (CONICET) por financiar este estudio en el marco de una Beca Doctoral Tipo I otorgada a VHZ.

\section{REFERENCIAS BIBLIOGRÁFICAS}

Araujo, C. O.; Condez, T. H. \& Sawaya, R. J. 2009. Anfíbios Anuros do Parque Estadual das Furnas do Bom Jesus, sudeste do Brasil, e suas relações com outras taxocenoses no Brasil. Biota Neotropica 9(2):77-98

ARZABE, C. 1999. Reproductive activity patterns of anurans in two different altitudinal sites within the Brazilian Caatinga. Revista Brasileira de Zoologia 16(3):851-864

ÁviLA, R. W. \& FerreIRA, V. L. 2004. Riqueza e densidade de vocalizações de anuros (Amphibia) em uma área urbana de Corumbá, Mato Grosso do Sul, Brasil. Revista Brasileira de Zoologia 21(4):887-892.

BARBAULt, R. 1991. Ecological constraints and community dynamics: linking community patterns to organismal ecology. The case of tropical herpetofaunas. Acta Oecologica 12(1):139-163.

BAsso, N. G. 1990. Estrategias adaptativas en una comunidad subtropical de anuros. Cuadernos de Herpetología, Serie Monografías 1:1-77.

Bellis, E. D. 1962. The influence of humidity on Wood Frog activity. American Midland Naturalist 68:139-148.

Bernarde, O. S. \& KoKubum, M. N. C. 1999. Anurofauna do município de Guararapes, Estado de São Paulo, Brasil (Amphibia: Anura). Acta Biologica Leopoldensia 21:89-97.

Bernarde, P. S. 2007. Ambientes e temporada de vocalização da anurofauna no Município de Espigão do Oeste, Rondônia, Sudoeste da Amazônia - Brasil (Amphibia: Anura). Biota Neotropica 7(2):87-92.

Blaustein, A. R.; Han, B. A; Relyea, R. A.; Johnson, P. T. J.; Buck, J. C.; GERVASI, S. S. \& KATS, L. B. 2011. The complexity of amphibian population declines: understanding the role of cofactors in driving amphibian losses. Annals of the New York Academy of Sciences 1223:108-119.

BorgeS, F. J. A. \& JULIANO, R. F. 2007. Distribuição espacial e temporal de uma comunidade de anuros do município de Morrinhos, Goiás,
Brasil (Amphibia: Anura). Neotropical Biology and Conservation 2(1):21-27.

Both, C.; Kaefer, I. L.; Santos, T. G. \& Cechin, S. T. Z. 2008. An austral anuran assemblage in the Neotropics: seasonal occurrence correlated with photoperiod. Journal of Natural History 42:205-222.

Cabrera, A. L. \& WillinK, W. 1980. Biogeografía de América Latina. Segunda edición corregida. Colección de Monografías Científicas de la Secretaría General de la Organización de los Estados Americanos. Washington D. C., Programa Regional de Desarrollo Científico y Tecnológico. 120p.

Cajade, R.; Schaefer, E. F.; Duré, M. I.; Kehr, A. I. \& Marangoni, F. 2010. Reproductive biology of Argenteohyla siemersi pederseni Williams and Bosso, 1994 (Anura: Hylidae) in northeastern Argentina. Journal of Natural History 44(31-32):1953-1978.

Canelas, M. C. S. \& Bertoluci, J. 2007. Anurans of the Serra do Caraça, southeastern Brazil: species composition and phenological patterns of calling activity. Iheringia, Série Zoologia 97:21-26.

Cardoso, A. J.; Andrade, G. V. \& Haddad, C. F. B. 1989. Distribuição espacial em comunidades de anfíbios (Anura) no sudeste do Brasil. Revista Brasileira de Biologia 49:241-249.

CARNEVALI, R. 2003. EI Iberá y su Entorno Fitogeográfíco. Corrientes, EUDENE. 112p.

Снао, A. 1987. Estimating the Population Size for Capture-Recapture Data with Unequal Catchability. Biometrics 43(4):783-791.

Colwell, R. K. 2009. EstimateS: Statistical estimation of species richness and shared species from samples. Version 8. Persistent URL. Available at < purl.oclc.org/estimates>. Accessed on May 2014.

Colwell, R. K. \& Coddington, J. A. 1994. Estimating terrestrial biodiversity through extrapolation. Philosophical Transactions of the Royal Society of London Series B 345:101-118.

Conte, C. E. \& Rossa-Feres, D. C. 2007. Riqueza e distribuição espaçotemporal de anuros em um remanescente da Floresta de Araucária no sudeste do Paraná. Revista Brasileira de Zoologia 24(4):1025-1037.

Duellman, W. E. 1978. The biology of an ecuatorial herpetofauna in Amazonian Ecuador. Miscellaneous Publications of the Museum of Natural History of the University of Kansas 65:1-352.

1999. Distribution patterns of amphibians in South America. In: Duellman, W. E ed. Patterns of distribution of amphibians: a global perspective. Baltimore, Johns Hopkins, p. 255-328.

Duellman, W. E. \& Koechlin, J. E. 1991. The reserva Cuzco Amazónico, Perú: Biological investigations, conservation, and ecoturismo. Occasional Papers of the Museum of Natural History of the University of Kansas 13:1-12.

ForTI, L. R. 2009. Temporada reprodutiva, micro-hábitat e turno de vocalização de anfíbios anuros em lagoa de Floresta Atlântica, no Sudeste do Brasil. Revista Brasileira de Zoociências 11(1):89-98.

FundaCión Biodiversidad. 2004. Fauna del Iberá: composición, estado de conservación y propuestas de manejo. Proyecto ARG 02/G35 GEF/PNUD/Fundación Ecos.101 p.

Gambale, P. G.; Woitovicz-Cardoso, M.; Vieira, R. R.; Batista, V. G.; Ramos, J. \& Bastos, R. P. 2014. Composição e riqueza de anfíbios anuros em remanescentes de Cerrado do Brasil Central. Iheringia, Série Zoologia 104(1):50-58.

Garey, M. V. \& DA SiLva, V. X. 2010. Spatial and temporal distribution of anurans in an agricultural landscape in the Atlantic Semideciduous Forest of southeastern Brazil. South American Journal of Herpetology 5(1):64-72.

Gordo, M. \& Campos, Z. 2005. Anuros das Serras de Entorno do Pantanal Sul. Corumbá, Embrapa Pantanal. 20p.

GrandinetTI, L. \& JACOBI, C. M. 2005. Distribuição estacional e espacial de uma taxocenose de anuros (Amphibia) em uma área antropizada em Rio Acima - MG. Lundiana 6:21-28.

GRÖMPING, U. 2006. relaimpo: Relative Importance of Regressors in Linear Models. R package version 1.1-1.

Haddad, C. F. B. \& Prado, C. C. 2005. Reproductive modes in frogs and their unexpected diversity in the Atlantic Forest of Brazil. Bioscience 55(3):207-217.

Hodgkison, S. \& Hero, J. 2001. Daily behavior and microhabitat use of the waterfall frog, Littoria nannotis in Tully Gorge, Eastern Australia. Journal of Herpetology 35(1):116-120. 
Ingaramo, M. R.; Etchepare, E. G.; Álvarez; B. B. \& Porcel, E. 2012 Riqueza y composición de la fauna de anuros en la región oriental de la Reserva Natural Provincial Esteros del Iberá, Corrientes, Argentina. Revista de Biología Tropical 60:759-769.

Kopp, K.; Signorelli, L. \& Bastos, R. P. 2010. Distribuição temporal e diversidade de modos reprodutivos de anfíbios anuros no Parque Nacional das Emas e entorno, estado de Goiás, Brasil. Iheringia, Série Zoologia 100(3):192-200.

Krebs, C. J. 1999. Ecological methodology. Menlo Park, Addison Wesley Educational Publishers. 620p.

Lindemann, R. H.; Merenda P. F. \& Gold, R. Z. 1980. Introduction to Bivariate and Multivariate Analysis. Glenview, Scott Foresman. 36p.

LiPs, K. R.; ReAser, J. K.; Young, B. E. \& IbÁÑez, R. 2001. Amphibian monitoring in Latin America: A protocol manual. Herpetological Circulars 30:1-116.

MARSH, D. M. 2001. Behavioral and demographic responses of Túngara frogs to variation in pond density. Ecology 82:1283-1292.

Martins, A.; Pontes, R.; Mattedi, C.; Fratani, J.; Murta-Fonseca, R. A.; Ramos, L. \& Brandão, A. L. R. 2014. Anuran community of a coastal Atlantic Forest fragment in the state of Rio de Janeiro, southeastern Brazil. Salamandra 50(1):27-39.

Melo, G. V.; Rossa-Feres, D. C. \& JiM, J. 2007. Variação temporal no sítio de vocalização em uma comunidade de anuros de Botucatu, Estado de São Paulo, Brasil. Biota Neotropica 7(2):93-102.

Moreno, C. E. 2001. Métodos para medir la biodiversidad. Zaragoza, MyT-Manuales \& Tesis SEA. 84p.

Narvaes, P.; Bertoluci, J. \& Rodrigues, M. T. 2009. Composição, uso de hábitat e estações reprodutivas das espécies de anuros da floresta de restinga da Estação Ecológica Juréia-Itatins, sudeste do Brasil. Biota Neotropica 9(2):117-123.

NeIFF, J. J. 2004. EI Iberá. ¿en peligro? Buenos Aires, Fundación Vida Silvestre de Argentina. 89p.

NeIfF, J. J. \& Poi de NeIfF, A. 2006. Situación ambiental en la Ecorregión Iberá. In: Brown, A.; Martinez Ortiz, U.; Acerbi, M. \& CorcherA, J. eds. La Situación Ambiental Argentina 2005. Buenos Aires, Fundación Vida Silvestre Argentina, p.177-184.

OdA, F. H.; Bastos, R. P. \& SÁ Lima, M. A. D. C. 2009. Taxocenose de anfíbios anuros no Cerrado do Alto Tocantins, Niquelândia, estado de Goiás: diversidade, distribuição local e sazonalidade. Biota Neotropica 9(4):219-232.

Palmer, M. W. 1990. The estimation of species richness by extrapolation. Ecology 71:1195-1198.

Pansonato, A.; Mott, T. \& Strüssmann, C. 2011. Anuran amphibians' diversity in a northwestern area of the Brazilian Pantanal. Biota Neotropica 11(4):77-86.

Pechmann, J. H. K.; Scott, D. E.; Semiltsch, R. D.; Caldwell, J. P.; Bit, L. J. \& GibBons, W. 1991. Declining amphibians populations: the problem of separating humans impacts from natural fluctuations. Science 253:825-940.

Perotti, M. G. 1997. Modos reproductivos y variables cuantitativas reproductivas de un ensamble de anuros del Chaco semiárido, Salta, Argentina. Revista Chilena de Historia Natural 70:277-288.

Prado, C. P. D. A.; Uetanabaro, M. \& Haddad, C. F. B. 2005. Breeding activity patterns, reproductive modes, and habitat use by anurans (Amphibia) in a seasonal environment in the Pantanal, Brazil. Amphibia-Reptilia 26(2):211-221.

Prado, G. M. \& Pombal, J. P. 2005. Distribuição espacial e temporal dos anuros em um brejo da Reserva Biológica de Duas Bocas, Sudeste do Brasil. Arquivos do Museu Nacional 63:685-705.

R CORE TEAm. 2015. R: a language and environment for statistical computing. R Austria, Foundation for Statistical Computing, Vienna. Available at $<$ https://www.r-project.org/> . Accessed on May 2014.
RöDel, M. \& ERnst, R. 2004. Measuring and monitoring amphibian diversity in tropical forests. An evaluation of methods with recommendations for standardization. Ecotropica 10:1-14.

Santos, T. G.; Kopp, K.; Spies, M. R.; Trevisan, R.; \& Cechin, S. Z. 2008. Distribuição temporal e espacial de anuros em área de Pampa, Santa Maria, RS. Iheringia, Série Zoologia 98(2):244-253.

Santos, T. G.; Rossa-Feres, D. C. \& Casatti, L. 2007. Diversidade e distribuição espaço-temporal de anuros em região com pronunciada estação seca do sudeste do Brasil. Iheringia, Série Zoologia 97(1):3749.

Schulze, A.; JANSEN, M. \& KöHLER, G. 2009. Diversity and ecology of anuran communities in San Sebastián (Chiquitano region, Bolivia). Salamandra 45(2):75-90.

Scott, N. J. \& Woodward, B. D. 1994. Surveys at breeding sites. In: Heyer, W. R.; Donnelly, M. A.; MCDiarmid, R. W.; Hayer, L. C. \& Foster, M. S. eds. Measuring and Monitoring Biological DiversityStandard Methods for Amphibians. Washington, Smithsonian Institution Press, p.118-125.

Sugai, J. L. M. M.; Terra, J. S. T. \& Ferreira, V. L. 2014. Anurans of a threatened savanna area in western Brazil. Biota Neotropica 14(1):1-9.

Toledo, L. F.; ZINA, J. \& HAdDAD, C. F. B. 2003. Distribuição espacial e temporal de uma comunidade de anfíbios anuros do Município de Rio Claro, São Paulo, Brasil. Holos Environment 3(2):136-149.

Uetanabaro, M.; Souza, F. L.; Landgref Filho, P.; Beda, A. F. \& Brandão, R. A. 2007. Anfíbios e répteis do Parque Nacional da Serra da Bodoquena, Mato Grosso do Sul, Brasil. Biota Neotropica 7(3):279-289.

VAIRA, M. 2002. Anurans of a subtropical montane forest in northwestern Argentina: ecological survey and a proposed list of species of conservation concern. Biodiversity and Conservation 11:1047-1062.

Vaira, M.; Akmentins, M.; Attademo, A.; Baldo, D.; Barrasso, D.; Barrionuevo, S.; Basso, N.; Blotto, B.; Cairo, S.; Cajade, R.; Céspedez, J.; Corbalán, V.; Chilote, P.; Duré, M.; Falcione, C.; Ferraro, D.; Gutierrez, F.; Ingaramo, M. R.; Junges, C.; Lajmanovich, R.; Lescano, J. N.; Marangoni, F.; Martinazzo, L.; Marti, R.; Moreno, L.; Natale, G. S.; Pérez Iglesias, J.; Peltzer, P.; Quiroga, L.; Rosset, S.; Sanabria, E.; Sanchez, L.; Schaefer, E.; Úbeda, C. \& Zaracho, V. 2012. Categorización del estado de conservación de los Anfibios de la República Argentina. Cuadernos de Herpetología 26:131-159.

VASCONCElOS, T. S. \& Rossa-FERES, D. C. 2005. Diversidade, distribuição espacial e temporal de anfíbios anuros (Amphibia, Anura) na região noroeste de estado de São Paulo, Brasil. Biota Neotropica 5:1-14.

Vieira, W. L. S.; Arzabe, C. \& Santana, G. G. 2007. Composição e distribuição espaço-temporal de anuros no Cariri Paraibano, nordeste do Brasil. Oecologia Brasiliensis 11(3):383-396.

Well, K. D. 1977. The social behaviour of anuran amphibians. Animal Behaviour 25(3):666-693.

Wilson, J. J. \& Maret, T. J. 2002. A comparison of two methods for estimating the abundance of amphibians in aquatic habitats. Herpetological Review 33(2):108-110.

Zaracho, V. H.; Ingaramo, M. R.; Semhan, R. V.; Etchepare, E. G.; Acosta, J. L.; Falcione, A. C. \& Álvarez, B. B. 2014. Herpetofauna de la Reserva Natural Provincial Isla Apipé Grande (Corrientes, Argentina). Cuadernos de Herpetología 28(2):153-160.

Zina, J.; Ennser, J.; Pinheiro, S. C. P.; Haddad, C. F. B. \& Toledo, L. F. 2007. Taxocenose de anuros de uma mata semidecídua do interior do Estado de São Paulo e comparações com outras taxocenoses do Estado, Brasil. Biota Neotropica 7:49-58. 